\title{
Titanium disilicide formation by wide-area electron beam irradiation
}

\author{
Cameron A. Moore, J. J. Rocca, and G. J. Collins \\ Department of Electrical Engineering and Engineering Research Center, Colorado State University, Fort \\ Collins, Colorado 80523
}

P. E. Russell and J. D. Geller
JEOL USA, Peabody, Massachusetts 01960

(Received 3 January 1984; accepted for publication 28 April 1984)

\begin{abstract}
We describe the use of a wide-area $\left(38 \mathrm{~cm}^{2}\right)$ electron beam as a heat source to interdiffuse $400-\AA$ thick sputter-deposited titanium films into $3-6-\Omega \mathrm{cm}\langle 100\rangle n$-type silicon wafers. Isochronal exposures of $30 \mathrm{~s}$ with electron beam of current densities greater than $250 \mathrm{~mA} / \mathrm{cm}^{2}$ reduced the as-deposited sheet resistance by a factor of 10 , while exposures at half this current caused the sheet resistance to increase by a factor of 2.5. Compositional depth profiles obtained from a combination of ion beam sputtering and Auger electron spectroscopy show that this resistivity increase is caused by diffusion of oxygen into the titanium film induced by the electron beam heating. At exposures to beam intensities sufficient to induce complete silicide formation, oxygen is segregated at the surface by the advancing silicon. We conclude that the silicide self-cleanses itself of oxygen during formation.
\end{abstract}

Silicides are attractive materials for both interconnections and contacts for very large scale integrated (VLSI) devices fabricated in silicon because of their low resistivities (as compared to highly doped polysilicon), their compatability with processing at high temperatures, and their controllable Schottky ohmic contact formation. ${ }^{.}$Of particular interest is titanium disilicide because its low resistivity $(\sim 15 \mu \Omega \mathrm{cm})$ at room temperature) lies below that of pure $\operatorname{Ti}(42 \mu \Omega \mathrm{cm})$, thus offering a lower contact resistance than pure Ti. Additionally, $\mathrm{TiSi}_{2}$ yields, as do most refractory metal silicides, barrier heights of nearly the same magnitude on both $n$ - and p-type silicon.

Silicides are generally created by one of two methods: (i) codeposition (via physical or chemical vapor deposition) of the constituent materials, usually requiring a subsequent high-temperature $\left(>800^{\circ} \mathrm{C}\right.$ ) annealing step to form the stoichiometric silicide, or (ii) deposition of the metallic film followed by a lower temperature $\left(\sim 600^{\circ} \mathrm{C}\right)$ alloying heat treatment which interdiffuses the silicon and metal species to form the silicide. When a silicide is formed by interdiffusion of a deposited metal film on silicon, a more atomically clean silicide/silicon interface often results, thereby minimizing contamination-induced contact resistance variations. The heat source typically used in batch processing is a furnace. Alternative heat sources include scanned $50-100-\mu \mathrm{m}$-diam continuous laser and electron beams, ${ }^{2}$ and exposure to the radiation from $100-\mu$ s arc discharges in argon. ${ }^{3}$ An advantage of electron beam driven alloying over laser-formed silicide layers is that the high optical reflectivity of most metals is not a factor. Hence an additional film above the metal is not required to increase energy coupling as in Ref. 2.

The wide-area $\left(38 \mathrm{~cm}^{2}\right)$ source used in this work allows for rapid silicide formation in a single wafer in-line system. Prior to our present work a pulsed electron beam 1 in. in diameter was used to form $\mathrm{MoSi}_{2}$ from molybdenum-silicon layered structure using a $25-\mathrm{J}$ glow discharge electron beam of $1 \mu$ s duration propagating in $10^{-5}$ Torr helium. ${ }^{4}$ In a second prior study, a raster-scanned electron beam was used to form $\mathrm{TiSi}_{2}$ from $\mathrm{Ti}$ which was deposited atop $\mathrm{Si}$ or poly-Si structures. ${ }^{5}$ An observation common to both Refs. 4 and 5 was that as the sample was heated the sheet resistivity first doubled while no silicide was formed. Further heating caused the resistivity to drop rapidly with a corresponding growth in the silicide thickness. An explanation of this resistivity behavior is offered herein in tandem with a demonstration of the self-cleaning of $\mathrm{TiSi}_{2}$ during its formation using a 7.0-cm-diam beam capable of producing $90 \mathrm{~W} / \mathrm{cm}^{2}$ of 3-7$\mathrm{keV}$ electrons on a continuous basis.

The broad-area electron beam is produced by a glow discharge in helium (1 Torr) and residual atmospheric constituents $\left(1 \mathrm{mTorr} \mathrm{N}_{2}-\mathrm{O}_{2}\right)$. This apparatus is described in greater detail elsewhere. ${ }^{6}$ The beam electrons in this source have energies between 3 and $7 \mathrm{keV}$, giving electron penetration depths of less than $5000 \AA$ in the materials studied herein. ${ }^{7}$ This characteristic surface heating is advantageous for the application studied. Although these electrons may have sufficient energy to cause radiation damage to underlying insulating films, it is possible that this detrimental aspect would be avoided via self-annealing due to the high temperature of the wafer at $e$-beam cessation.

The samples used were formed on in situ, precleaned 3$6-\Omega \mathrm{cm}\langle 100\rangle n$-type $\mathrm{Si}$ wafers by sputter deposition of

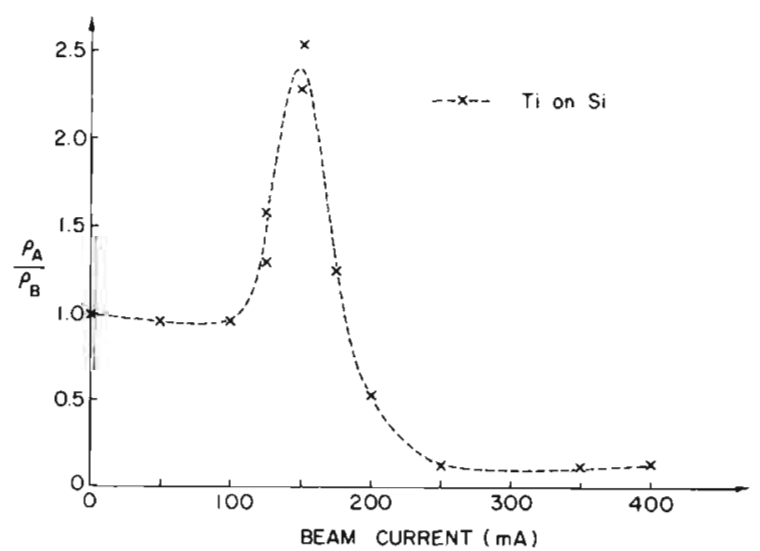

FIG. 1. Normalized resistance of titanium on silicon films as a function of beam current. Exposures for $30 \mathrm{~s}$ in 1 Torr helium. 

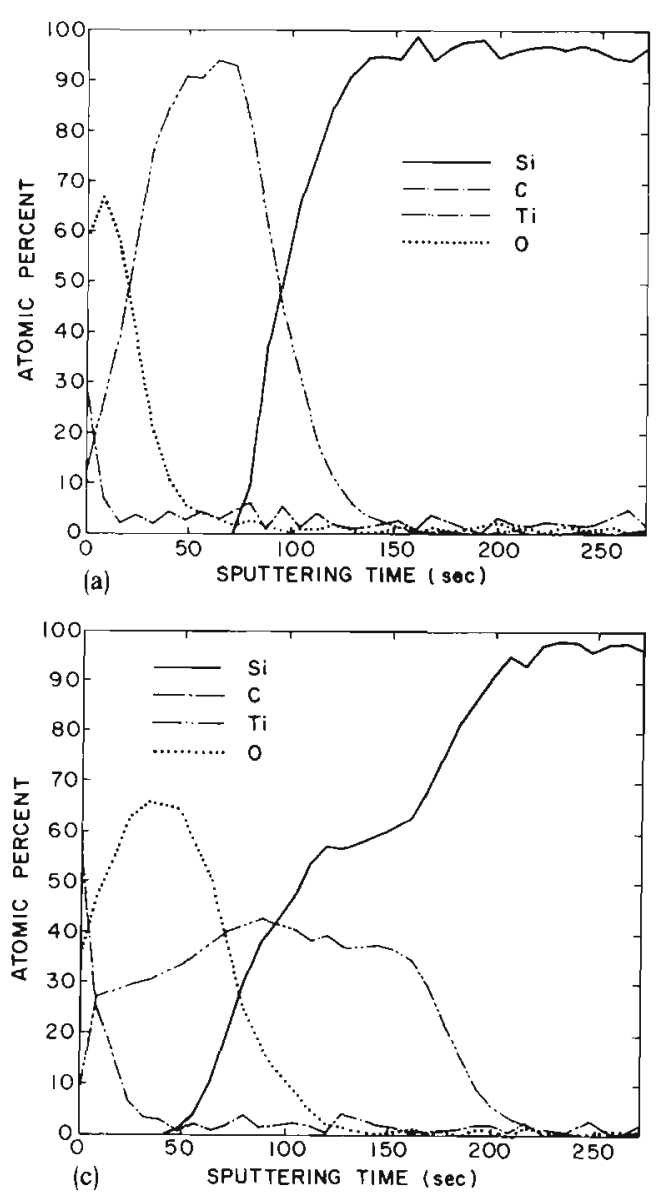

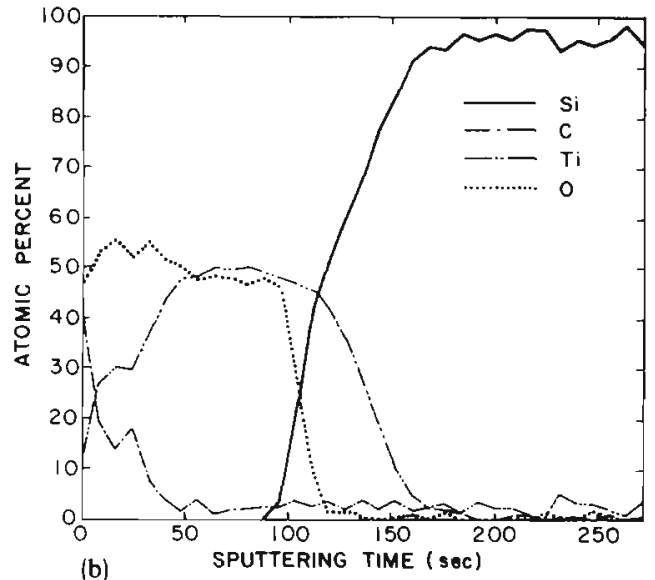

(b)

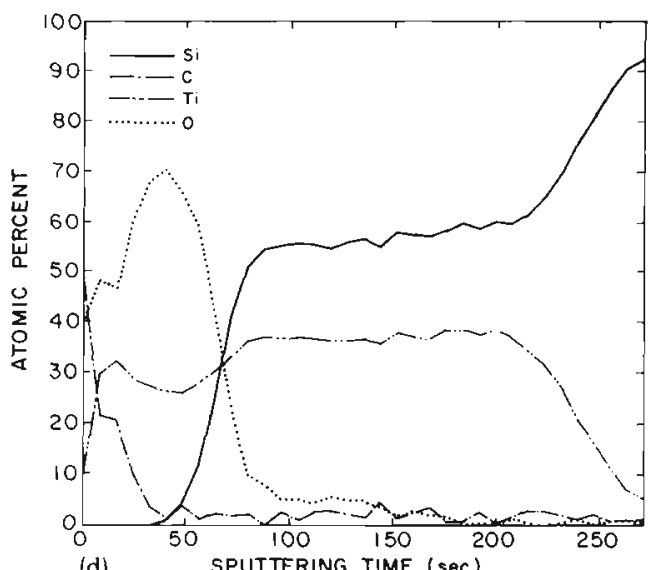

(d)

FIG. 2. Sputtered Auger depth profiles showing $\mathrm{Si}, \mathrm{Ti}, \mathrm{O}$, and $\mathrm{C}$ content. (a) As-deposited film; (b) film of resistance ratio $\sim 2.5$; (c) film of resistance ratio $\sim 1.0$; (d) film of resistance ratio $\sim 0.1$.

$\sim 400-\AA$ titanium films at $10 \AA / \mathrm{s}$ in a base pressure of $1.6 \times 10^{-7}$ Torr. The wafers were then cleaved into rectangular $1 \times 2 \mathrm{~cm}$ samples for measurement of sheet resistivities using a linear four-point probe and subsequent electron beam processing. The as-deposited samples had a resistance range of $21 \pm 2 \Omega / \square$. All samples were exposed to the electron beam for $30 \mathrm{~s}$. Though the formation reaction takes place in times much less than this, a 30-s exposure duration was employed so as to allow repeatable exposure conditions principally due to the manual ramp-up of the applied power to the desired level. The ratio $\left(\rho_{A} / \rho_{B}\right)$ of post- to pretreatment resistances helps to minimize geometry-dependent factors. This ratio as a function of electron beam discharge current is shown in Fig. 1. For exposures $\geqslant 250 \mathrm{~mA}$ the resistance ratio approached 0.1 , corresponding to a resistivity of $<20 \mu \Omega \mathrm{cm}$. The data corresponding to an electron beam current of $150 \mathrm{~mA}$ showed an increase of $\sim 2.5$ in resistivity, a phenomenon which has been observed before as a function of sintering temperature ${ }^{1}$ and time into an electron beam exposure. ${ }^{5}$ A study of film composition versus depth was undertaken to gain insight into this phenomenon.

To determine the relative depth profiles of constituent species of these samples, ion beam sputtering combined with Auger electron spectroscopy was performed. The species (and respective peaks) detected were $\mathrm{Si}(\mathrm{LMM}), \mathrm{Ti}(\mathrm{LMM})$, $\mathrm{O}(\mathrm{KLL})$, and $\mathrm{C}(\mathrm{KLL})$. Argon ions from $3 \mathrm{keV}, 0.6 \mu \mathrm{A}$ beam of $100 \mu \mathrm{m}$ diameter were raster scanned to etch over a 500 $\times 500 \mu \mathrm{m}$ area. The electron beam used to excite the Auger transitions had an energy of $10 \mathrm{keV}$, a current of $0.5 \mu \mathrm{A}$, and a diameter of $30 \mu \mathrm{m}$. The Auger data were collected for $20 \mathrm{~s}$ following an 8-s asynchronous ion bombardment etch. To make the comparisons regarding film composition which ensue, a similar processing history of the samples must be ensured. To achieve this a $4 \times 1 \mathrm{~cm}$ sample was processed such that its major axis extended from the center to the perimeter of the discharge, where it was clamped in place to a heat sink. In this way a temperature gradient along the length of the sample is induced during the electron beam heating. Via spatial measurement of the resistance ratio and calibration to Fig. 1 the electron beam current that gives equivalent heating was inferred. Though this indicates the beam's uniformity is lacking, uniform processing is still possible at properly chosen exposure conditons. ${ }^{6}$ Figure $2(a)$ shows the as-deposited film $\left(\rho_{A} / \rho_{B}=1.0\right)$ while Figs. $2(\mathrm{~b}), 2(\mathrm{c})$, and 2(d) correspond to electron beam exposures of 150,200 , and $250 \mathrm{~mA}$ and resistance ratios $\left(\rho_{A} / \rho_{B}\right)$ of $2.5,0.75$, and 0.1 , respectively. As shown in Fig. 2(b), an increase in carbon and oxygen incorporation in the film occurs.

For a moderate beam exposure $(150 \mathrm{~mA})$ oxygen diffuses through the Ti film, and roughly one-half the titanium becomes bonded to oxygen; the electrical conductivity is thus reduced by the same factor $(\sim 2)$. It was previously suggested that the increase in resistance was due to diffusion of $\mathrm{O}$ and/or Si into the $\mathrm{Ti}^{8}{ }^{8}$ Because in Fig. 2(b) there is negligi- 
ble Si transport, we conclude the sheet resistance increase to be caused solely by $\mathrm{O}$ diffusion. Heating from a higher beam current (200 mA) causes a thin $\mathrm{TiSi}_{2}$ film to form deep in the film while the advancing Si plows the incorporated oxygen towards the surface. Finally, in Fig. 2(d), the silicide film is completely formed when the requisite temperature is achieved and the impurities are principally in a surface layer which can be etched with high selectivity. The increase in oxygen and carbon in Fig. 2(b) is thought due to the poor vacuum used, though they are not as abundant in the completed film [Fig. 2(d)]. For this reason the He-air mixture (1 Torr/ $1 \mathrm{mTorr}$ ) was all we required.

The question remains whether Figs. 2(a)-2(d) are a sequential process. Since the heats of formation for oxides of titanium are greater than that for $\mathrm{TiSi}_{2},{ }^{9}$ it would be thermodynamically unlikely for the silicide to self-clean. This then would imply that the competition between $\mathrm{O}$ and $\mathrm{Si}$ in reaction with Ti favors the former at lower temperatures and the latter at higher temperatures. But this further implies a sample heated to obtain high resistance ratio could not subsequently be formed into $\mathrm{TiSi}_{2}$. This was disproven when a high resistance sample (previously treated at $150 \mathrm{~mA}$ for 30 s) was subsequently heated using $250 \mathrm{~mA}$ of electron beam current for $30 \mathrm{~s}$ and indeed the resistance ratio dropped to 0.1 . In light of this result we conclude the silicide self-cleans via advancement of underlying $\mathrm{Si}$.

In summary, we have achieved the formation of titanium disilicide films via wide-area electron beam alloying of $\mathrm{Ti}$ on $\mathrm{Si}$. These films formed by interdiffusion of $\mathrm{Ti}$ and $\mathrm{Si}$ exhibited an increase in resistance due to oxygen incorporation and bonding as oxides of titanium. However, when proper temperature-time cycling occurs, the oxygen impurities are swept entirely to the surface by the advancing silicon and an intermetallic-semiconductor interface of improved cleanliness is achieved. The wide-area electron beam used in this study is easily scaled to process samples $10 \mathrm{~cm}$ or more in diameter at unfocused power densities of up to $90 \mathrm{~W} / \mathrm{cm}^{2}$

The authors wish to thank H. K. Park of Tektronix Research Laboratories for providing the samples used in this work. This work was supported by the Office of Naval Research and the Air Force Office of Scientific Research.

'S. P. Murarka, J. Vac. Sci. Technol. 17, 775 (1980).

${ }^{2}$ T. Shibata, T. W. Sigmon, J. L. Regolini, and J. F. Gibbons, J. Electrochem. Soc. 128, 637 (1981).

${ }^{3}$ Juh Tzeng Lue, Yuen Chung Liu, and Wei Jiun Shen, Appl. Phys. Lett. 38, 372 (1981).

${ }^{4}$ Setsu Suzuki, Yasushi Ohkubo, Fumitomo Matsuoka, and Tadatsugu Itoh, Appl. Phys. Lett. 42, 797 (1983).

${ }^{5}$ E. A. Maydell, R. E. Harper, A. Abid, P. L. F. Hemment, and K. G. Stephens, Materials Research Society Annual Meeting, Boston, MA, Nov. 14-17, 1983 (Symposia in press).

${ }^{6}$ Cameron A. Moore, J. J. Rocca, T. Johnson, G. J. Collins, and P. E. Russell, Appl. Phys. Lett. 43, 290 (1983).

${ }^{7}$ CRC Handbook of Spectroscopy, edited by J. W. Robinson (CRC, Cleveland, 1974), Vol. 1.

${ }^{8}$ S. P. Murarka and D. B. Fraser, J. Appl. Phys. 51, 342 (1980).

${ }^{\circ}$ JANA Thermochemical Tables, second edition, edited by D. R. Stull and H. Prophet (NSRDS-National Bureau of Standards, Washington, D. C., 1971), p. 37.

\title{
Characteristics of amorphous silicon staggered-electrode thin-film transistors
}

\author{
M. J. Powell and J. W. Orton \\ Philips Research Laboratories, Redhill, Surrey, United Kingdom
}

(Received 19 March 1984; accepted for publication 1 May 1984)

\begin{abstract}
Amorphous silicon staggered-electrode thin-film transistors (TFT's) can show current crowding near the origin in the output characteristics. The degree of current crowding is governed by the voltage dependence of the current flowing from the $n^{+}$contact to the conducting channel. This current is a space-charge-limited current whose magnitude depends on the bulk density of states in the undoped intrinsic layer. For a $0.5-\mu \mathrm{m}$-thick $i$ layer, calculations predict negligible current crowding for $N(E)<10^{16} \mathrm{~cm}^{-3} \mathrm{eV}^{-1}$, but severe current crowding for $N(E)>3 \times 10^{16}$ $\mathrm{cm}^{-3} \mathrm{eV}^{-1}$. Experimental results are consistent with $N(E)$ in the range $10^{16} \mathrm{~cm}^{-3} \mathrm{eV}^{-1}-2 \times 10^{16}$ $\mathrm{cm}^{-3} \mathrm{eV}^{-1}$. This is lower than the value derived from the transfer characteristic of the TFT $\left(\sim 10^{17} \mathrm{~cm}^{-3} \mathrm{eV}^{-1}\right)$, which is evidence for an inhomogeneous distribution of deep gap states through the $0.5-\mu \mathrm{m}$ film of $\alpha-\mathrm{Si}: \mathrm{H}$.
\end{abstract}

There is considerable interest in the use of amorphous silicon thin-film transistors (TFT's) for matrix addressing large area liquid crystal displays, and prototype TFT addressed liquid crystal display panels have recently been demonstrated. ${ }^{1.2}$ The TFT characteristics required depend on the detailed specification of the display (TV, alpha-numeric, size, resolution, etc.). However, it is generally desirable for them to show good switching ratio and high "on current" at modest switching voltages.

It is also important that an adequate "on current" is 